\title{
DIE PALÄOGEOGRAPHIE UND FAZIEN DER KAMBRISCHEN ABLAGERUNGEN IM BEREICH DER OSTEUROPÄISCHEN PLATTFORM
}

\author{
VINCAS KORKUTIS
}

VINCAS KORKUTIS, 1984: Die Paläogeographie und Fazien der kambrischen Ablagerungen im Bereich der osteuropäischen Plattform. Bull. Geol. Soc. Finland 56, Part 1-2, 189-201.

Die kambrischen Ablagerungen im baltischen Gebiet sind weit verbreitet und sie bestehen aus marinen - terrigenen Sedimenten, die bestimmte charakteristische paläontologische Merkmale haben (Tafel 1 und 2). Die Mächtigkeit der Ablagerungen übersteigt $250 \mathrm{~m}$.

Die meisten unterkambrischen Ablagerungen (Mobergella, Holmia und Protolenus Zonen) sind im westbaltischen Gebiet verbreitet (Abb. 1). Die lithologisch-paläontologische Karte zeigt folgende drei Zonen: Zone 1 ist aus Sandsteinen bis zu $85 \%$ und Aleuroliten bis zu $15 \%$ zusammengesetzt; Zone 2 aus Sandsteinen $30-60 \%$, Aleuroliten $5-40 \%$ und Tonen 5-20\%; Zone 3 aus Tonen bis zu $65 \%$, Aleuroliten $20-35 \%$ und Sandsteinen $5-15 \%$. Die Mächtigkeit der Ablagerungen reicht bis zu etwa $100 \mathrm{~m}$ (Zemyté - $80 \mathrm{~m}$, Jagodnaja - $107 \mathrm{~m}$ ).

Die mittelkambrischen Ablagerungen (Paradoxides oelandicus und P. paradoxissimus Zonen) sind im west- und mittelbaltischen Gebiet verbreitet (Abb. 2). Die lithologisch-paläontologische Karte zeigt vier Zonen: Zone 1 ist aus Sandsteinen bis zu $95 \%$ und Aleuroliten bis zu 5-15\% zusammengesetzt; Zone 2 aus Sandsteinen bis zu 50\%, Aleuroliten 5-30\% und Tonen 5-15\%; Zone 3 aus Tonen bis zu $50 \%$, Aleuroliten 5-25\% und Sandsteinen 5-15\%, Zone 4 aus Tonen bis zu $65 \%$, Aleuroliten 25-35\% und Sandsteinen 5-10 \%. In einigen Bohrungen wurden Karbonate bis zu 3-5\% gefunden. Die Mächtigkeit der Ablagerungen reicht bis zu $150 \mathrm{~m}$ (Zemyté - $109 \mathrm{~m}$, Jagodnaja - $159 \mathrm{~m}$ ).

\section{ABSTRACT}

Cambrian deposits composed of marine - terrigenous sediments having paleontologic characteristics are widely distributed throughout the Baltic region (Tables 1 and 2). The thickness of the Cambrian deposits exeed $250 \mathrm{~m}$.

The majority of the Cambrian deposits (of the Mobergella, Holmia and Protolenus zones) occur in western Baltic regions (Fig. 1). The paleogeographic map shows three lithological facies zones: 1) with up to $85 \%$ sandstones and up to $15 \%$ aleurolites; 2) up to 30-60\% sandstones, 5-40\% aleurolites and 5-20\% clays; 3) up to $65 \%$ clays, $20-35 \%$ aleurolites and $5-15 \%$ sandstones. The deposits may be as much as $100 \mathrm{~m}$ thick (Ľemyte $-80 \mathrm{~m}$, Jagodnaja $-107 \mathrm{~m}$ ).

The Middle Cambrian deposits (of the Paradoxides oelandicus and P. paradoxissimus zones) are distributed in the western and central Baltic regions (Fig. 2). Four lithological facies zones can be distinguished on the paleogeographic map: 1) with up to $95 \%$ sandstones and $5-15 \%$ aleurolites; 2) up to $50 \%$ sandstones, $5-30 \%$ aleurolites and $5-15 \%$ clays; 3 ) up to $50 \%$ clays, $5-25 \%$ aleurolites and 5-15\% sandstones; 4) clays up to $65 \%, 25-35 \%$ aleurolites and 5-10\% sandstones. Rocks containing up to $3-5 \%$ carbontates have been found in some drill holes. The deposits are up to $150 \mathrm{~m}$ thick (Ľemyte $-109 \mathrm{~m}$, Jagodnaja $159 \mathrm{~m})$.

Key words: Cambrian, Baltic region, paleogeography, fossils.

V. Korkutis: Lithuanian Geological Research and Survey Institute, Sevcenkos 13, 232600 Vilnius, Lithuania (USSR). 


\section{Einleitung}

Die Ablagerungen des Kambriums sind im ganzen Gebiet Litauens weit verbreitet. Sie bestehen aus terrigenen Bildungen mariner und schelfischer Herkunft, die eine Mächtigkeit von über $250 \mathrm{~m}$ haben können. An den Grenzen der Republik findet man kambrische Ablagerungen unter einer dicken Decke paläozoischer, mesozoischer und känozoischer Schichten, wo sie nur mit Hilfe von Tiefbohrungen erreichbar sind. Sie liegen in 200 bis $2100 \mathrm{~m}$ Tiefe.

Das erste Tiefbohrloch in Vilnius wurde im Jahre 1948 gebohrt, und damals wurden die unterkambrischen und mittelkambrischen Ablagerungen sowie das kristallische Fundament erreicht. Seither sind neue Bohrungen in anderen Gebieten Litauens gemacht worden, die viel neues geologisches Material über die Paläographie und die Fazien der kambrischen Ablagerungen ergaben. Später zeigte sich, dass die Sedimentgesteine in den westlichen und in den östlichen Teilen Litauens verschiedenes Alter haben, was sich durch die ungleiche tektonische Geschichte der geologischen Entwicklung dieser Regionen erklären lässt. Als Erster hat V. Korkutis (1966) hierauf aufmerksam gemacht. Er hat mit Hilfe von detaillierten lithologischen und paläontologischen Forschungen bewiesen, dass die kambrischen Bildungen in den westlichen Gebieten Litauens jünger sind als die »blauen Schlicke» der östlichen Gebiete. Später hat V. Korkutis (1968, 1971, 1972 und 1981) eingehend auch die wesentlichen Schnitte des kambrischen Systems beschrieben und die Reste der Makrofauna gesammelt und erforscht.

Die tektonische Struktur des südbaltischen Gebiets (Mazurische Hebung) bedingte die Verschiedenheit der kambrischen Ablagerungen und ihre Verteilung in verschiedene fazielic Zonen. Im westlichen Teil Litauens findet man die vollständigsten Schnitte, die seine mittlere Zone charakterisieren. Das Zentrum Litauens liegt im östlichen Teil der Baltischen Syneklise, in der die kambrischen Schnitte unvollständig sind. Die dritte strukturfazielle Zone liegt in den östlichen Gebieten Litauens, wo die für die Moskau-Syneklise charakteristischen Gesteine verbreitet sind.

Die kambrischen Ablagerungen im Baltikum werden aufgrund ihrer lithologisch-faziellen Merkmale und der paläontologischen Charakteristik stratigraphisch eingeteilt in unterkambrische (Subholmia-, Mobergella-, Holmia- and Protolenus-Zonen) und mittelkambrische ( $\mathrm{Pa}$ radoxides-Zone) Ablagerungen.

\section{Unterkambrische Ablagerungen}

Die ältesten Gesteine des Unterkambriums sind im östlichen Teil des Baltischen Beckens verbreitet und lagern diskordant auf den tiefer liegenden Vendbildungen. Sie gehören zur baltischen Serie der Osteuropäischen Tafel und korrelieren mit Sedimenten der Moskau-Syneklise. Der Lontowa-Horizont-Stratotyp kommt in Nordestland nicht weit von der Stadt Kunda vor und ist 74,3 m mächtig (Mens, Pirrus 1977). In Litauen füllt der »blaue Schlick» die breite Dzükijes-Biegung, die am Rande der MoskauSyneklise entlanggeht. Die Mächtigkeit der Decke ist sehr verschieden und variiert zwischen 204 m (Bohrung Pǒskos) und 978 m (Bohrung Pliavins, Sowjetrepublik Lettland). Die Mächtigkeit der kambrischen Sedimente ist in den einzelnen Bohrungen verschieden und variiert von ein paar Meter bis zu 115,5 m in der Bohrung Tverečius.

Die baltische Serie lagerte sich in einem flachen marinen Becken ab, als die östlichen und nördlichen Gebiete des Baltikums allmählich untertauchten. Vor der Lontowa-Zeit transgressierte das Meer in Form von Buchten von der Moskau-Syneklise her. Sonst war die baltische Region Festland mit schwach differenziertem Relief. Davon zeugen die Verwitterungskruste im Hangenden der Vend- und älteren Bildungen sowie auch die verwitterten Oberflächen in der Basis der baltischen Serie. 
Die klastischen Sedimente wurden aus den denudierten Gebieten gebracht, die nord- und westwärts von dem Sedimentationsbecken der unterkambrischen Periode lagen.

Die Zwischenlagen von Konglomeraten und schrägschichtigem Sandstein im »blauen Schlick», die Trockenrisse und Wellenrippeln zeugen davon, dass sich das terrigene Material in einem seichten marinen Becken abgelagert hat. Das Becken wurde ostwärts tiefer, weil die Menge des klastischen terrigenen Materials des Lontowa-Horizonts gleichmässig von Osten nach Westen zunimmt.

Aus den abgelagerten blaugrünen und grüngrauen Sedimenten in dem dort anzutreffenden Pyrit darf man schliessen, dass das Milieu in dem marinen Becken im früheren Kambrium labil und reduziert war. Im Lauf der einzelnen Zeitperioden wechselte dieses Milieu zwischen oxydiert und reduziert, darum findet man in den Profilschnitten die glaukonitischen Zwischenlagen. Das Klima war wahrscheinlich ziemlich warm und feucht (V. Korkutis u.a., 1972; Aren B. u.a. 1979; Brangulis A., 1979; Makhnach A., 1958, 1970; Tynni R., Donner J., 1980; Tynni R., 1982; Vidal G., 1981).

Die organische Welt im Meer war nicht reich. Hier lebten Anneliden, Brachiopoden und Akritarchen (Sokolov B., 1974; Timofejev B. und andere, 1976).

Die Basis der Schichtenfolge besteht aus dunkelgrauem geschichtetem Ton mit dichtgepackten Sandsteinen. Die Sandsteine sind feinkörnig, quarzig, und sie enthalten Feldspat, Pyrit, Glimmer und Glaukonit. Oben auf den Sandsteinen lagern graugrüne, plastische, feinschichtige, glimmerhaltige Tone mit mehreren Sandsteinzwischenschichten, die mit Pyrit und Glaukonit bereichert sind. Bei der Analyse der Thermokurve wurde festgestellt, dass das Grundmineral der Tone Hydroglimmer aus dem Lontowa-Horizont ist. Die Tone haben oft eine Beimischung von Kaolinit. Im mittleren Teil des Profils sind die Tone farbig: grüngrau, rotviolett und gelblichbraun. In dieser Schicht findet man reichliche Reste von Anneliden: Sabellidites cambriensis Yan., Yanichevskyites petropolitanus (Yan.) Sok., Platysolenites antiquissimus Eichw. Von Akritarchen Granomarginata prima Naum., Gr. squamacae Volk., Tasmanites tenellus Volk., Leiomarginata simplex Naum., Leiosphaeridia sp.

Die Ablagerungszonen Mobergella, Holmia und Protolenus liegen nur in den westlichen Gebieten des Baltischen Beckens. Unterkambrische Bildungen findet man in vollständigen Schnitten, welche auf dem kristallischen Fundament lagern. In manchen Gebieten im Osten lagern unterkambrische Schichten diskordant auf der Verwitterungskruste der »blauen Schlicke». Nach der Erforschung der AisčiuSerie teilte V. Sakalauskas (1966) sie in drei Schichtenfolgen ein, nämlich Gegés, Virbalio und Kybartu.

Die erste Schichtenfolge, die Gegés-Suite, besteht aus dunkelgrauen und graugrünen Argilliten, die manchmal mit hellen Sandsteinen und Aleuroliten abwechseln. Bezeichnend für die Schichtenfolge sind braune und dunkelgrüne, eisenhaltige Oolithzwischenlagen mit vergrösserter Eisenmenge. In manchen Zwischenlagen ist Glaukonit enthalten. Als Stratotyp der ersten Schichtenfolge ist das Profil der Bohrung Kybartai-22 in 1361-1390 m Tiefe erklärt worden. Sie hat eine Mächtigkeit bis zu $29 \mathrm{~m}$.

In den westlichsten Schnitten der Bohrung Salantai-1 kommen grüngraue Argillite und Tone vor mit 6-7 Zwischenlagen von glaukonitischen Sandsteinen und eisenhaltigen Oolithen. Die mineralogischen Analysen ergaben, dass die Zwischenlagen, die eine grössere Eisenmenge haben, aus Quarzkörnern von 0,1 bis $1 \mathrm{~mm}$ Grösse mit Einmischung grober Fraktien bestehen. Der Zement ist dunkelbraun göthithämatitisch oder grün und dunkelgrün chamositisch. In allen Zeitperioden sind Sandsteine mit Texturen vom »Kråksten»-Typus verbreitet. Überall sieht man Spuren der im flachen Meer stattgehabten Sedimentation, schrägwellige 

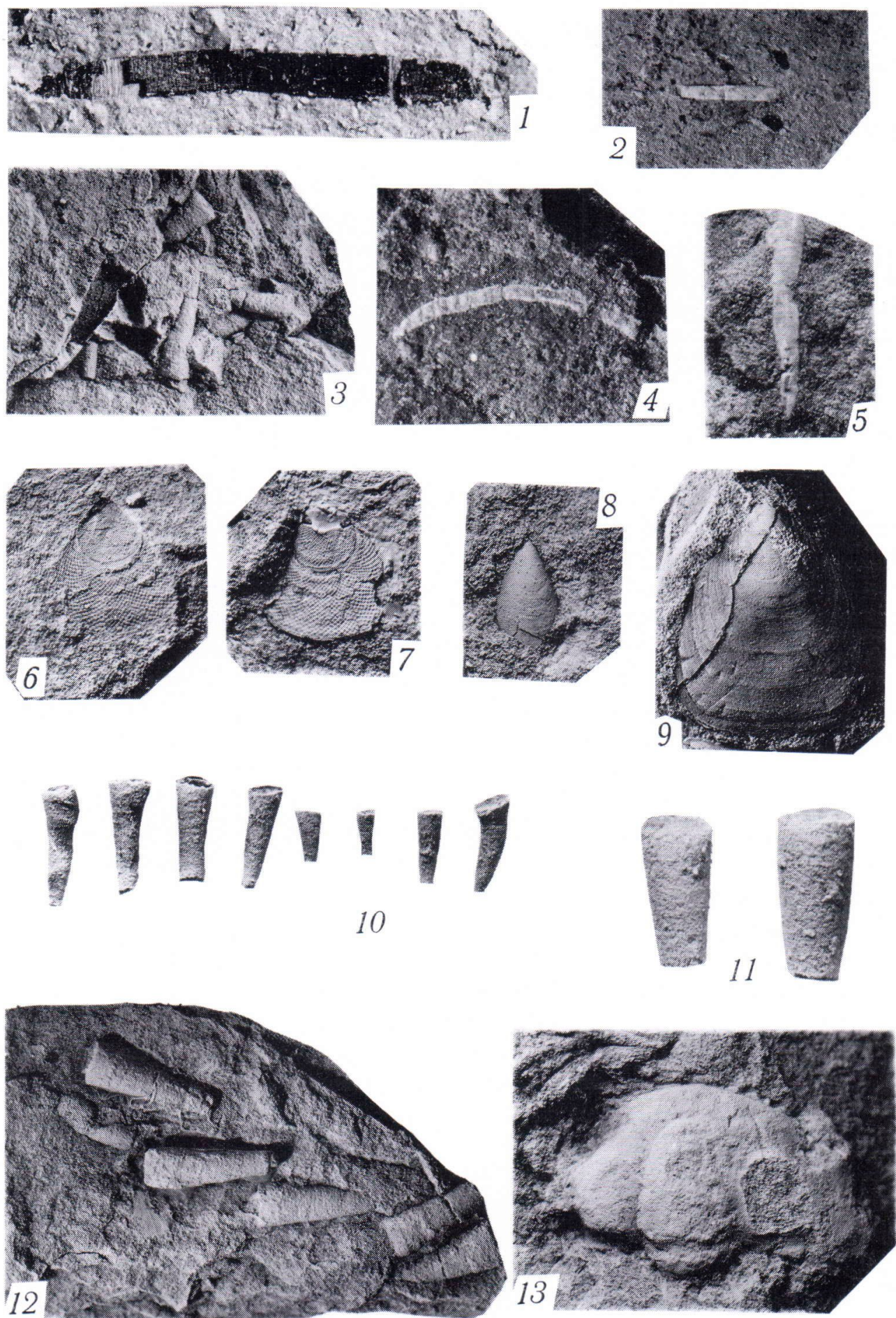
Tafel I

Fig. 1. Sabellidites cambriensis Yanichevskyj. Unterkambrium, Zone Subholmia, Osteuropäische Plattform. 1- Die allgemeine Gestalt des Wurmes mit klarer Segmentation, Litauische SSR, Bohrung Vilnius, Tiefe 364.0 m. Nr. 23a-8, $\times 10$.

Fig. 2. Yanichevskyites petropolitanus (Yan.) Sokolov. Unterkambrium, Zone Subholmia, Osteuropäische Plattform. 2Die allgemeine Gestalt des Wurmes, Estnische SSR, Bohrung Chirvli, Tiefe 137.2 m. Nr. 76-8, × 3 .

Fig. 3. Volborthella tenuis Schmidt. Unterkambrium, Zone Holmia, Osteuropäische Plattform. 3- Massenanhänfung von Muscheln und Kernen, Lettische SSR, Bohrung Pavilosta, Tiefe 1423.95 m. Nr. 91-8, $\times 3$.

Fig. 4. Yanichevskyites petropolitanus (Yan.) Sok. Unterkambrium, Zone Holmia, Osteuropäische Plattform. 4- Die allgemeine Gestalt des Wurmes, Lett. SSR, Bohrung Pavilosta, Tiefe 1423.90 m. Nr. 75-8, $\times 4$.

Fig. 5. Hyolithellus sp. Unterkambrium, die Zone Holmia, Osteuropäische Plattform, 5- Die allgemeine Gestalt, Lett. SSR, Bohrung Kolka, Tiefe 851.9-852.0 m. Nr. 83/3-8, × 5 .

Fig. 6-7. Acrothelle bellapunctata Walcott. Unterkambrium, Zone Holmia, Osteuropäische Plattform. 6- Abdruck der Klappe, Lett. SSR, Bohrung Pavilosta, Tiefe 1378.4 m. Nr. 360-8, × 3. 7- Ventralklappe, Lett. SSR, Pavilosta, Tiefe 1378.2 m. Nr. $359-8, \times 3$.

Fig. 8. Westonia finlandensis Walcott. Unterkambrium, Zone Protolenus, Osteuropäische Plattform. 8- Ventralklappe, Lett. SSR, Pavilosta, Tiefe 1342. 3 m. Nr. 357-8, × 3 .

Fig. 9. Lingulella nathorsti Linnarsson. Unterkambrium, Zone Protolenus, Osteuropäische Plattform. 9- Ventralklappe, Lett. SSR, Stirnas, $1147.1 \mathrm{~m} . \mathrm{Nr} .141-8, \times 3$.

Fig. 10-12. Volborthella tenuis Schmidt. Unterkambrium, Zone Protolenus, Osteuropäische Plattform. 10- Die allgemeine Gestalt der Muscheln. Lett. SSR, Bohrung Pavilosta, Tiefe 1367.85 m. Nr. 85-8, × 3. 11- Die allgemeine Gestalt zweier Muscheln, Litauische SSR, Bohrung Šakiai, 1430.65 m. Nr. 80a-8, × 3. 12- Ansamlung von Muschelkernen, Lett. SSR, Bohrung Pavilosta, Tiefe 1354.50 m. Nr. 84-8, $\times 2$.

Fig. 13. Strenuella insolita N. Tschernyscheva. Unterkambrium, Zone Protolenus, Osteuropäische Plattform. 13- Kranidium, Lett. SSR, Bohrung Pavilosta, Tiefe 1329.5 m., $\times 3$.

Table I

Fig. 1. Sabellidites cambriensis Yanichevskyj. Sub-holmia stage, Lower Cambrian, East-European platform. 1- General view of a worm with a distinct segmentation, Lithuanian SSR, Vilnius $364.0 \mathrm{~m}$. No. 23a-8, $\times 10$.

Fig. 2. Yanichevskyites petropolitanus (Yan.) Sokolov. Sub-holmia stage, Lower Cambrian, East-European platform. 2General view of a worm. Estonian SSR, Chirvli $137.2 \mathrm{~m}$. No. 76-8, $\times 3$.

Fig. 3. Volborthella tenuis Schmidt. Holmia stage, Lower Cambrian, East-European platform. 3- Group of shells and piths. Latvian SSR, Pavilosta 1423.95 m. No. 91-8, × 3.

Fig. 4. Yanichevskyites petropolitans (Yan.) Sok. Holmia stage, Lower Cambrian, East-European platform. 4- General view of a worm. Latvian SSR, Pavilosta $1423.90 \mathrm{~m}$. No. $75-8, \times 4$.

Fig. 5. Hyolitellus sp. Holmia stage, Lower Cambrian, East-European platform. 5- General view. Latvian SSR, Kolka $851.9-852,0$ m. No. $83 / 3-8, \times 5$.

Fig. 6-7. Acrothelle bellapunctata Walcott. Holmia stage, Lower Cambrian, East-European platform. 6- Fossil impression of a shell. Latvian SSR, Pavilosta 1378.4 m. No. 360-8, $\times 3$. 7 - Ventralside of a shell, Latvian SSR, Pavilosta $1378.2 \mathrm{~m}$. Nr. 359-8, × 3 .

Fig. 8. Westonia finlandensis Walcott. Protolenus Zone, East-European platform. 8- Ventralside of a shell, Latvian SSR, Pavilosta 1342.3 m. No. 357-8, × 3 .

Fig. 9. Lingulella nathorsti Linnarsson. Protolenus Zone, Lower Cambrian, East-European platform. 9- Ventralside of a shell, Latvian SSR, Stirnas $1147.1 \mathrm{~m}$. No. $141-8, \times 3$.

Fig. 10-12. Volborthella tenuis Schmidt. Protolenus Zone, Lower Cambrian, East-European platform. 10- General view of shells, Latvian SSR, Pavilosta $1367.85 \mathrm{~m}$. No. 85-8, × 3. 11- General view of two shells, Lithuanian SSR, Šakiai 1430.65 m. No. 80a-8, × 3. 12- Group of shellpiths, Latvian SSR, Pavilosta 1354.50 m. No. 84-8, $\times 2$.

Fig. 13. Srenuella insolita N. Tschernyscheva. Protolenus Zone, Lower Cambrian, East-European platform. 13- Cephalon, Latvian SSR, Pavilosta $1329.5 \mathrm{~m} . \times 3$ 

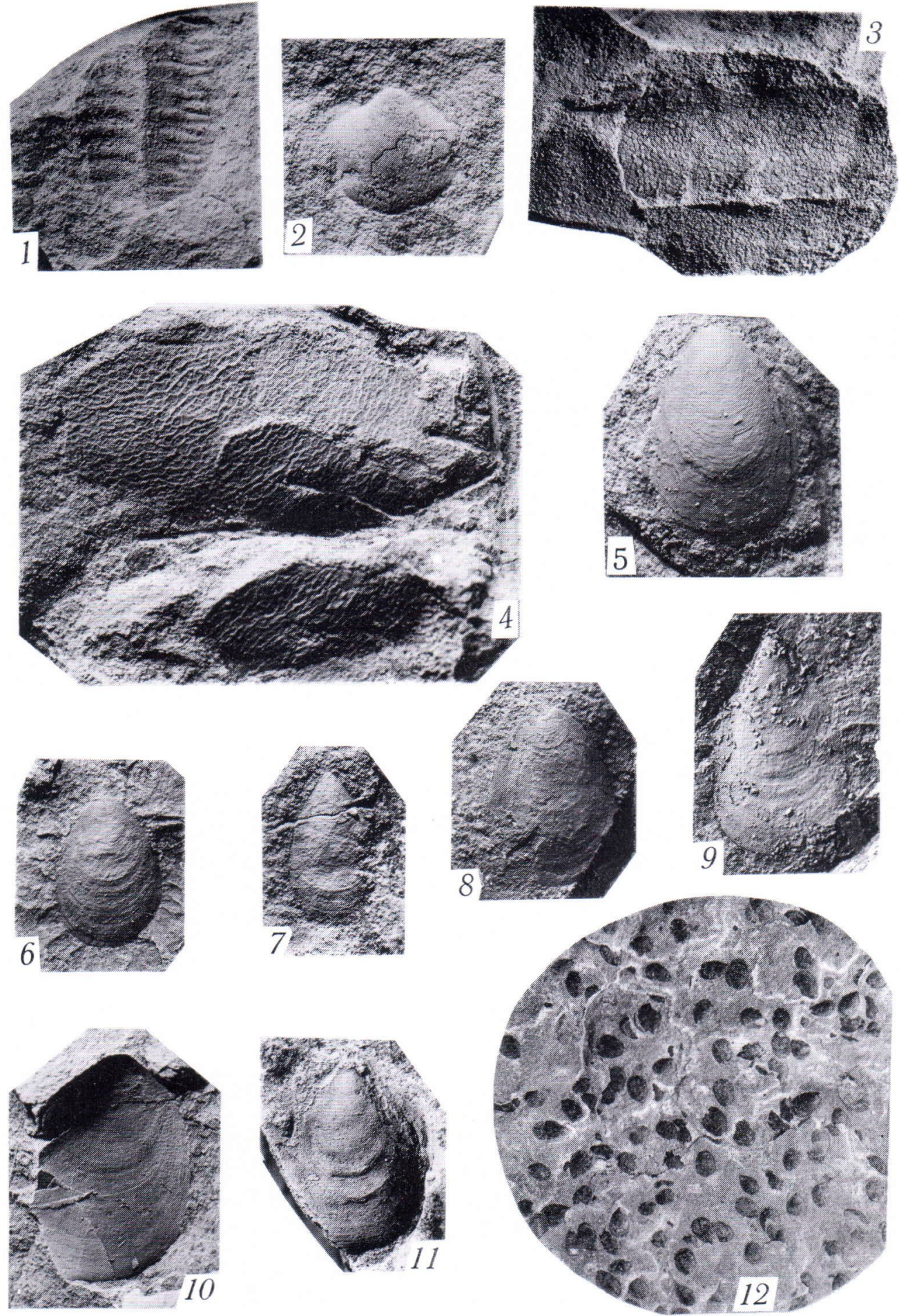
Tafel II

Fig. 1. Strenuella insolita N. Tschernyscheva. Unterkambrium, Zone Protolenus. 1 - Abdruck des Körpers, Lett. SSR, Bohrung Pavilosta, Tiefe $1312.5 \mathrm{~m} . \times 3$, koll. K. Mens.

Fig. 2. Ellipsocephalus sp. Unterkambrium, Zone Protolenus Osteuropäische Plattform. 2- Kranidium, Litauische SSR, Bohrung Gargždai - 4, Tiefe $2112.0 \mathrm{~m} . \times 3$.

Fig. 3-4. Ceratiocaris sp. Unterkambrium, Zone Protolenus Osteuropäische Plattform. 3- Gestalt mit klarer Segmentation, Litauische SSR, Bohrung Žarenai, Tiefe 2013.0 m. Nr. 176a-8, × 2.5. 4- Gestalt mit klarer Segmentation, Lit. SSR, Bohrung Gargždai -4 , Tiefe 2108.5 m. Nr. 158-8, × 5 .

Fig. 5. Lingulella sp. Unterkambrium, Zone Protolenus Osteuropäische Plattform. 5- Ventralklappe, Lett. SSR, Bohrung Stirnas, Tiefe $1140.0 \mathrm{~m} . \mathrm{Nr} .138-8, \times 5$.

Fig. 6. Lingulella desiderata (Walcott). Mittelkambrium Zone Paradoxides Osteuropäische Plattform. 6- Dorsalklappe, Lit. SSR, Bohrung Plungé - 3, Tiefe 1875.4 m. Nr. 151/8-8, × 5 .

Fig. 7-9. Lingulella ferruginae Salter. Mittelkambrium, Zone Paradoxides Osteuropäische Plattform. 7- Ventralklappe, Lit. SSR, Bohrung Kranžanté, Tiefe 1706.7 m. Nr. 191-8, × 5. 8- Dorsalklappe, Lit. SSR; Bohrung Kražanté, Tiefe 1710.2 m. Nr. 196-8, × 5. 9- Ventralklappe, Lit. SSR, Bohrung Kražanté, Tiefe 1713.05 m. Nr. $198-8, \times 5$.

Fig. 10. Westonia finlandensis Walcott. Mittelkambrium, Zone Paradoxides Osteuropäische Plattform. 10- Dorsalklappe, Lit. SSR, Bohrung Plungé -3 , Tiefe 1875.4 m. Nr. $151 / 2 \mathrm{a}-8, \times 5$.

Fig. 11. Lingulella agnostorum Wallerius. Mittelkambrium, Zone Paradoxides Osteuropäische Plattform. 11- Dorsalklappe, RTFSR, Bohrung Gusev - 2, Tiefe 1615.9 m. Nr. 117-8, × 3 .

Fig. 12. Lingulella ferruginea Salter. Mittelkambrium, Zone Paradoxides Osteuropäische Plattform. 12- Massenanhäufung von Ventral- und Dorsalklappen, Lit. SSR, Bohrung Kražantié, Tiefe 1712.8 m. Nr. 195/1-8, Natürliche Grösse.

Table II

Fig. 1. Strenuella insolita N. Tschernyscheva. Protolenus Zone, Lower Cambrian, 1- Fossil impression of thorax, Latvian SSR, Pavilosta $1312.5 \mathrm{~m}$. × 3. Coll. by K. Mens.

Fig. 2. Ellipsocephalus sp. Protolenus Zone, Lower Cambrian, East-European platform. 2- Cephalon, Lithuanian SSR, Gargždai $-4,2112.0 \mathrm{~m} . \times 3$.

Fig. 3-4. Ceratiocaris sp. Protolenus Zone, Lower Cambrian, East European platform. 3- View with a distinct segmentation, Lithuanian SSR, Žarenai 2013.0 m. No. 176a-8, × 2.5. 4- View with a distinct segmentation, Lithuanian SSR, Gargždai $-4,2108.5$ m. No. $158-8, \times 5$.

Fig. 5. Lingulella sp. Protolenus Zone, Lower Cambrian, East-Europaean platform. 5- Ventral side of a shell, Latvian SSR, Stirnas $1140.0 \mathrm{~m}$. No. $138-8, \times 5$.

Fig. 6. Lingulella desiderata (Walcott). Paradoxides Zone, Middle Cambrian, East-European platform. 6- Dorsal side of a shell, Lithuanian SSR, Plungé $-3,1875.4$ m. No. 151/8-8, ×5.

Fig. 7-9. Linguella ferruginea Salter. Paradoxides Zone, Middle Cambrian, East-European platform. Ventral side of a shell, Lithuanian SSR, Kražanté, 1706.7 m. No. 191-8, × 5. 8- Dorsal side of a shell, Lithuanian SSR, Kražanté,

1710.2 m. No. 196-8, × 5. 9- Ventral side of a shell, Lithuanian SSR, Kražanté, 1713.05 m. No. 198-8, $\times 5$.

Fig. 10. Westonia finlandensis Walcott. Paradoxides Zone, Middle Cambrian, East-European platform. 10- Dorsal side of a shell, Lithuanian SSR, Plungé $-3,1875.4 \mathrm{~m}$. No. $151 / 2 \mathrm{a}-8, \times 5$.

Fig. 11. Lingulella agnostorum Wallerius. Paradoxides Zone, Middle Cambrian, East-European platform. 11- Dorsal side of a shell, RTFSR, Gusev - 2, 1615.9 m. No. $117-8, \times 3$.

Fig. 12. Lingulella ferruginea Salter. Paradoxides Zone, Middle Cambrian, East-European platform. 12- Group of ventral and dorsal sides of shells. Lithuanian SSR, Kražanté, 1712.8 m. No. 195/1—8, normal size. 
Schichtung mit Wellenrippeln und Trockenrissen. In der Gegés-Suite wurden Spuren von Zephalopoden: Volborthella tenuis Schm. (Bohrung Šakiai-42, 1430,65 m Tiefe), von Krustentieren: Ceratiocaris sp. (Bohrung Gargždai-4, 2108,5 m Tiefe) und von Brachiopoden: Lingulella sp. gefunden. In der westlichen Schichtenfolge fanden sich Reste von Trilobiten: Germaropyge? mendosa Tch. (Bohrung Gargždai-6, 2095,5 m Tiefe) und Ellipsocephalus sp. (Bohrung Gargždai-4, $2112 \mathrm{~m}$ ). In Lettland sind viel Volborthella tenuis Schm., Luekatiella sp., Hyolithellus sp. gefunden worden. Der in der Schichtenfolge Gegés-Suite angetroffene Akritarchenkomplex zeigt, dass sie ins untere Kambrium gehören.

Die zweite Schichtenfolge - Virbalio-Suite - ist an der gleichen Stelle wie die Schichtenfolge Gegés verbreitet, und sie lagert auf ihr mit allmählichen Übergängen.

Die Virbalio-Suite ist in den westlichen Gebieten mehr tonig und besteht aus Aleuroliten, seltener aus graugrünen sandig - aleurolitischen Argilliten und grüngrauen Tonen. Im oberen Teil dominieren die graugrünen Aleuroliten mit Zwischenlagen von hellgrauen Sandsteinen. Glaukoniten (bis $12 \%$ ) und Brachiopodentrümmern. Im östlichen Teil Litauens ist die Schichtenfolge Virbalio-Suite sandiger.

Der Parastratotyp der Schichtenfolge Virbalio-Suite liegt in der Bohrung Kybartai-22 in 1322-1361 m Tiefe. Er besteht aus für die frag- liche Schichtenfolge typischen Gesteinen, die auf dem Stratotyp der Gegés-Suite lagern. Hinsichtlich der sedimentfaziellen und lithologischen Zusammensetzung unterscheidet sich die Virbalio-Suite von dem früheren Meer insofern, als sich hier keine eisenreichen Sedimente ablagerten. Alle anderen physiogeographischen Bedingungen waren ähnlich. In den westlichen Gebieten des Baltischen Beckens lagerte sich graugrüner, toniger Schlamm ab. Nordwärts (in Richtung des heutigen Baltischen Schildes), südwärts (in Richtung der Masurischen $\mathrm{He}$ bung) und westwärts (in Richtung des ehemaligen Fenno-Sarmatischen Randes) sieht man den faziellen Übergang der tonigen Sedimente in die psammitischen Ablagerungen. Das marine Bekken war seicht, weil man überall in den Schichten sandige Zwischenlagen trifft, die tonhaltigen, feinen, syngenetischen Quarz führen. Das Becken wurde nach Westen hin tiefer, weil in dieser Richtung der Tongehalt und die gesamte Mächtigkeit der Gesteine zunehmen. In den Gesteinen der Virbalio-Suite finden sich Brachiopoden: Westonia $\mathrm{cf}$. finlandensis Walcott (Bohrung Jurbarkas, 1480,1 m und 1538,4 m Tiefe; Bohrung Plungé, 1875,4 m), Westonia cf. elongatus Walcott (Lettland, Bohrung Pavilosta, 1335,02 m), ferner Trilobiten: Strenuella (Comluella) insolita N. Tchern (Lettland, Bohrung Pavilosta, 1324,0 m 1307,0 m), Ellipsocephalus sp.

Die paläogeographische Karte (Abb. 1) zeigt

Abb. 1. Lithologisch-paläogeographische Karte der unterkambrischen Ablagerungen im Baltikum

1. Zone von reichen Sandsteinen (bis $85 \%$ ) und Aleuroliten (bis $15 \%$ ).

2. Zone von Sandsteinen $(60-30 \%)$, Aleuroliten $(40-5 \%)$ und Tonen $(20-5 \%)$.

3. Zone von Tonen (bis $65 \%$ ), Aleuroliten (35-20\%) und Sandsteinen $(15-5 \%$ ).

4. Hauptsächliche Bruchlinien.

5. SW-Grenze der Osteuropäischen Plattform.

6. Grenze der heutigen Verbreitung der unterkambrischen Ablagerungen.

7. Die Richtung der Meeresregression.

8. Wahrscheinliche Isopachen.

Fig. 1. The lithologic-palaeogeographic map of the Lower Cambrian of the Baltic basin. 1: Zone of sandstones (up to $85 \%$ ) and aleurolites (up to $15 \%$ ). 2: Zone of sandstones $(60-30 \%)$, aluerolites $(40-5 \%)$ and clays $(20-5 \%)$. 3: Zone of clays (up to $65 \%$ ), aleurolites $(35-20 \%)$ and sandstones $(15-5 \%)$. 4: Main fractures, 5 : Southwestern boundary of the East European platform. 6: Boundary of present distribution of deposits. 7: Land relatively uplifted and direction of removal. 8: Supposed isopachytes. 


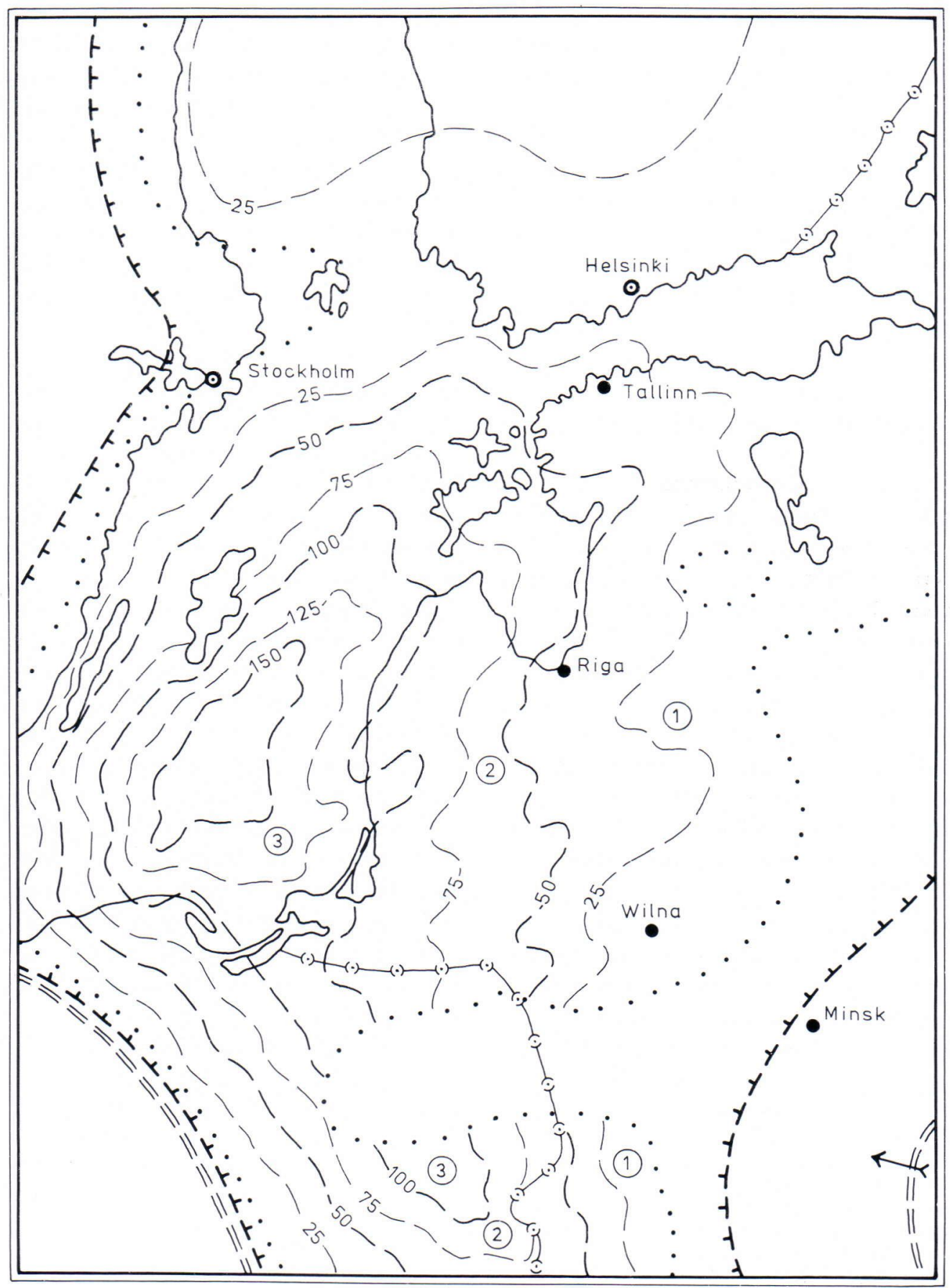

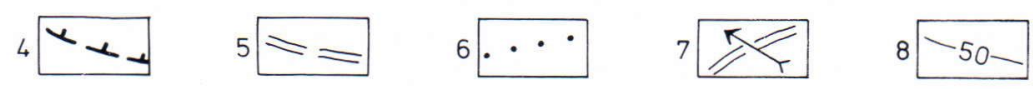


die unterkambrischen Zonen (Mobergella, Holmia und Protolenus). Zone 1 ist zusammengesetzt aus Sandsteinen bis $85 \%$ und Aleuroliten bis $15 \%$; Zone 2 aus Sandsteinen $60-30 \%$, Aleuroliten $40-5 \%$ und Tonen $20-5 \%$; Zone 3 aus Tonen bis $65 \%$, Aleuroliten 20$35 \%$ und Sandsteinen 15-5 \%. Unterkambrische Ablagerungen sind weitverbreitet und gut korreliert in Polen und Schweden (Lendzion K., 1968, 1976; Moczydlowska M., 1981, Vidal G., 1981).

\section{Mittelkambrische Ablagerungen}

Die dritte mittelkambrische Schichtenfolge Kybartai-Suite lagert mit allmählichen Übergängen auf der Virbalio-Suite. Sie besteht aus dunklen Argilliten, Aleuroliten und Zwischenlagen von hellen Sandsteinen mit Glaukonit. Die Schichtung der Gesteine ist horizontal, seltener wellig und sehr selten linsenförmig. Die Zwischenlagen von Argilliten sind mächtiger und dominieren im Schnitt. In manchen Bohrungen haben sie eine Mächtigkeit bis zu einigen Metern und führen keine Sandsteine.

Das Becken blieb noch in der Zeitperiode der Virbalio-Suite bestehen, es war aber noch flacher, und überall lagerten sich sandige Küstenbildungen ab. Auch die Fläche des Beckens wurde kleiner - die Regression breitete sich in den zentralen Gebieten Estlands, sowie in den östlichen Gebieten Lettlands und Litauens aus. Die hydrodynamischen Verhältnisse des Bekkens Kybartai-Suite waren stiller, weil es hier kaum typische »Kråksten»-Texturen gibt. Das
Klima war wahrscheinlich feucht und verhältnismässig warm. In diesen Schichten gibt es viele Brachiopoden - Lingulella cf. ferruginea Salt., L. cf. desiderata Walc., L. cf. agnostorum Wall., L. lithuanica Korkutis.

Der Parastratotyp der Schichtenfolge Kybartai-Suite liegt in der Bohrung Kybartai-22 in 1306,9-1322,3 m Tiefe. In diesem Intervall findet man den entsprechenden Akritarchenkomplex. Diese Ablagerungen repräsentieren die Zone Paradoxides oelandicus.

Das Profil des Kambriums im Baltischen Becken endigt mit mächtigen Massen von Sandsteinen, die V. Sakalauskas (1966) als DeimenosSerie unterscheidet. Als Stratotyp bezeichnet er die Bohrung Pravdinsk -1 in 1852,4-1936,0 m Tiefe. Höher lagern die paläontologisch gut charakterisierten Bildungen des Ordoviciums, unten die Gesteine der Kybartai-Suite.

Die Deimenos-Serie ist charakterisiert von weissen, quarzischen Sandsteinen und dunkelgrauen, glimmerigen Argilliten. Die Sandsteine haben gute gemeinsame Merkmale, von feinkörnigen bis $\mathrm{zu}$ verschiedenkörnigen. Als Parastratotyp der Schichtenfolge DeimenosSerie können die Ablagerungen in der Bohrung Kybartai-22 in 1279,6-1306,9 m gelten.

Während der Sedimentation der DeimenosSerie war die baltische Region der Rand des mächtigen Fenno-Sarmatischen Kontinents, der teilweise vom seichten epikontinentalen Meer überflutet war. Im marinen Becken des Mittelkambriums lagerten sich weisse, quarzische Sandsteine $a b$. Im allgemeinen sind Zwischenlagen von dunklen, bitumhaltigen Tonen im Schnitt selten, aber ihre Menge nimmt

Abb. 2. Lithologisch-paläogeographische Karte der mittelkambrischen Ablagerungen im Baltikum

1. Zone von reichen Sandsteinen (bis $95 \%$ ) und Aleuroliten (bis $15 \%$ ).

2. Zone von Sandsteinen (bis $50 \%$ ), Aleuroliten $(30-5 \%)$ und Tonen $(15-5 \%$ ).

3. Zone von Tonen (bis $50 \%$ ), Aleuroliten (25-5\%) und Sandsteinen (15-5\%).

4. Zone von Tonen (bis $65 \%$ ), Aleuroliten (35-25\%), Sandsteinen (10-5 \%) und Karbonaten (bis $5 \%$ ).

Fig. 2. The lithologic-palaeogeographic map of the Middle Cambrian of the Baltic basin. 1: Zone of sandstones (up to $95 \%$ ) and aleurolites (up to $15 \%$ ). 2: Zone of sandstones (up to $50 \%$ ), aleurolites $(30-5 \%)$ and clays $(15-5 \%$ ). 3 : Zone of clays (up to $50 \%$ ), aleurolites $(25-5 \%$ ) and sandstones (15-5\%). 4: Zone of clays (up to $65 \%$ ), aleurolites $(35-25 \%)$ and sandstones $(10-5 \%)$, carbonates (to $5 \%$ ). 


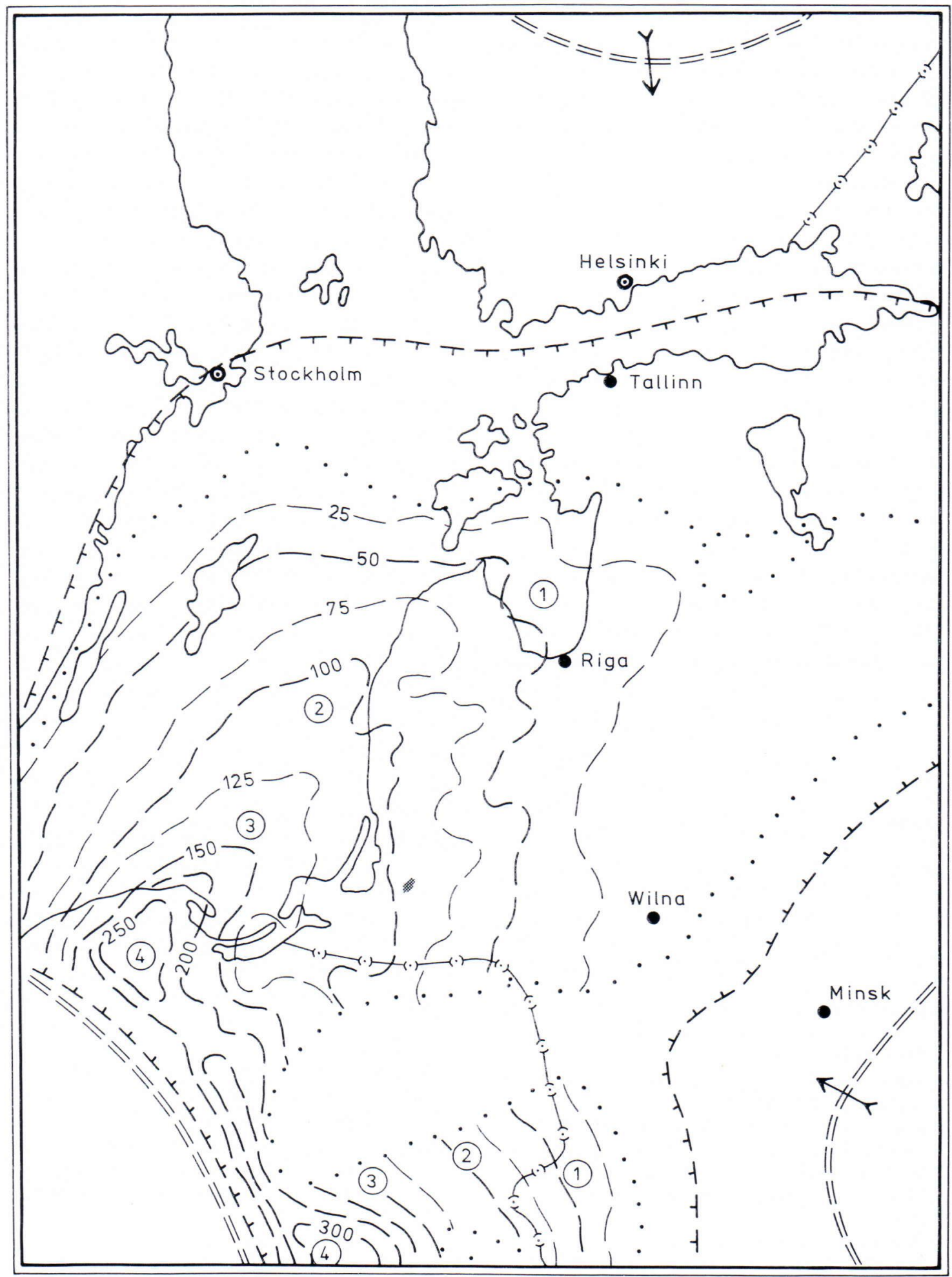


westwärts, in Richtung des Tieferwerdens des Beckens zu. Man trifft dünne Zwischenlagen von weissen Tonen. Der kaolinitische Zement der Sandsteine zeugt davon, dass in dem Becken manchmal die Verwitterungskruste in Form von Kaolin abgelagert wurde.

Ausgangs des Mittleren Kambriums brach die Meeresverbindung, die zu Beginn der unterkambrischen Zone Holmia-Protolenus hier entstanden war, ab. Die ganze baltische Region wurde zu einem kontinentalischen Festland, wo Denudationsprozesse vorherrschten.

Die paläographische Karte (Abb. 2) zeigt die mittelkambrischen Zonen (Paradoxides oelandicus und $P$. paradoxissimus). Zone 1 ist zusammengesetzt aus Sandsteinen bis $95 \%$ und Aleuroliten bis 15-5 \%; Zone 2 aus Sandsteinen bis $50 \%$, Aleuroliten 30-5 \% und Tonen 15$5 \%$; Zone 3 aus Tonen bis $50 \%$, Aleuroliten 25-5 \% und Sandsteinen 15-5\%; Zone 4 aus Tonen bis $65 \%$, Aleuroliten 35-25\%, Sandsteinen 10-5 \% und Karbonaten bis $5 \%$.

Die mittelkambrischen Sandsteine wurden von V. Korkutis $(1971,1976)$ in drei Schichten- folgen eingeteilt (von unten nach oben), nämlich Vilkyčiai-Suite, Šiupariai-Suite und DegliaiSuite. Die Vilkyčiai-Sandsteine haben eine Mächtigkeit von $10 \mathrm{~m}$ bis $18 \mathrm{~m}$ in Gargždai-6, und in der Bohrung Jagodnaja bis $30 \mathrm{~m}$. Die Šiupariai-Suite besteht aus aleurolitischen Tonen und Sandsteinen, in der Bohrung Degliai-2 $9 \mathrm{~m}$ mächtig, in der Bohrung Gargždai$618 \mathrm{~m}$ und im Profil Jagodnaja 28,5 m. Die Degliai-Suite besteht aus weissen, mittel- und feinkörnigen Sandsteinen mit einer Mächtigkeit von $37 \mathrm{~m}$ im Profil Degliai-2, $25 \mathrm{~m}$ in Gargždai-6 und bis 43,5 $\mathrm{m}$ in der Bohrung Jagodnaja-1.

$\mathrm{Zu}$ Beginn des oberen Kambriums war die baltische Region wie ein kontinentalisches Festland mit nur kurzen Transgressionen. In dem geosynklinischen Becken Westeuropas, in Nordpolen und in den benachbarten Regionen lagerten sich aleuritische Tone und Muschelkalke ab mit guten Brachiopoden und Trilobiten, die in der Bohrung Žarnowec eine Mächtigkeit bis $10 \mathrm{~m}$ haben.

\section{Literatur}

Aren, B.; Brangulis, A.; Volkova, N.; Lendizion, K.; Mens, K.; Michniak, R.; Paskeviciené, L.; Pirrus, E.; Rozanow, A. \& Yankauskas, T., 1979. Baltyiskaja synekyza. In: Stratigrafiya dokembriyskich i kembriyskich otlezhenij zapada Vostozhno - Europeiskoj platformy. Moscva, Nauka. 42-68.

Brangulis, A. P., 1979. Kembrijskaja systema. In: Geologizheskoje stroenije i poleznyje iskopaemyje Latviji Ryga, Zinatne. 18-38.

Korkutis, V. A., 1966. Trubzhatye zhervi nizhnego kembriya Juzhnoj Pribaltiki. In: Paleontologiya i stratigrafiya Pribaltiki i Belorusii. I(VI) Vilnius, Mintis. 7-29.

—, 1968. Stratigrafiya kembrijskich otlozhenij Juzhnoj Pribaltiki. In: Stratigrafiya nizhnego paleozojya Pribaltiki i korelyatsiya s drugimi Regionami. Vilnius, Mintis. $53-68$.

—, 1971. Kembriskie otlozheniya Bailtiskogo basseina. Vilnius, Mintis. $176 \mathrm{~S}$.

-, 1975. O konglomerate nizhnego kembriya Baltiskogo basseina i ego faune. In: Fauna i stratigrafiya paleozoiya i mesozoiya Pribaltiki i Belorussii. Vilnius, Mintis. 205-217.

—, 1976. Fauna kembrijskich otlozhenyj Juzhnoj Pribaltiki i stratigraphyzheskoje zhazhenije. In: Materialy po stratigraphyi Pribaltiki. Vilnius. 23-25.

-, 1981. Late Precambrian and Early Cambrian in the East European Platform. Precambrian Research, vol 15, $75-94$.

—, Lapinskas, P. P. \& Lashkov, E. M., 1972. Lithologiya i fatsii neftenosnych otlozhenij nizhnego paleozoya Juzhnoj Pribaltiki. Moscow, Nedra. 179 S.

Lendzion, K., 1968. Razvitie i stratigrafiya kembriya severo — vostozhnoj Polshe. In: Stratigrafiya nizhnego paleozoiya Pribaltiki i korreliaciya s drugimi Regionami. Vilnius, Mintis. 69--80.

-, 1976. Stratigrafia kambru zachodniej czesci syneklizy Perybaltyckiej. Biul. 270. In: $\mathrm{Z}$ badan geologicznych 
Nizu Polskiego, t. II, Biuletyn 270, Institut geologiczny. 59-84.

Makhnach, A. S., 1958. Drewnepaleozoiskyje otlozheniya Belorussij. Izd. Akad. Nauk. BSSR. Minsk. 225 S.

Makhnach, A. S., Bessonova, V. Y. \& Veretennikov, N. V., 1970. Stratigrafizheskaya schema verchnego dokembriya Belorussii i smezhnych raionov. Dokl, Akad. Nauk. BSSR. 14, 150-153.

Mens, K. \& Pirrus, E., 1977. Stratigraficheskije razrezy kembriya Estonii. Tallin, Valgus. $68 \mathrm{~S}$.

Moczydlowska, M., 1981. Lower and Middle Cambrian acritarcs from northeastern Poland. Precambrian Research, vol. 15, 63-74.

Sakalauskas, V. F., 1966. Novyje dannyje po stratigrafizheskoj scheme kembriskich otlozhenij Juzhnoj Pribaltiki. In: Tezisy dokl. XIX nauzhn. studenzheskoj konferenzij. Vilnius, Universitet.
Sokolov, B S., 1974. Periodichnost (etapnost) razvitija organiche - skogo mira i biostratigraficheskie granitsy. Geol. Geofiz. 3-9.

Timofejev, B. V., German, T. N. \& Michailova, N. S., 1976. Mikrofitofossilii dokembrija, kembrija i ordovika. Leningrad, Nauka. 1-107.

Tynni, R., 1982. New results of studies on the fossils in the Lower Cambrian sediment deposits of the Söderfjärden Basin. Bull. Geol. Soc. Finland, Nr. 54. 57-68.

Tynni, R. \& Donner, J., 1980. A microfossil and sedimentation study of the Late Precambrian formation of Hailuoto, Finland. Geol. Surv. of Finland, Bull 311. 35 p.

Vidal, G., 1981. Lower Cambrian acritarch stratigraphy in Scandinavia. Geol. För. Stockholm Förh. 103. 183-192.

Manuscript received, January 9, 1984. 